\title{
Coronavirus disease 19 and scientific publications
}

\author{
Francisco J. Bosques-Padilla ${ }^{1 *}$ and David Gómez-Almaguer ${ }^{2}$ \\ ${ }^{1}$ Gastroenterology Services; ${ }^{2 H e m a t o l o g y ~ S e r v i c e, ~ S c h o o l ~ o f ~ M e d i c i n e, ~ “ D r . ~ J o s e ́ ~ E l e u t e r i o ~ G o n z a ́ l e z ” ~ U n i v e r s i t y ~ H o s p i t a l, ~ U n i v e r s i d a d ~ A u t o ́ n o m a ~ d e ~}$ \\ Nuevo León, Monterrey, Nuevo León, Mexico
}

The coronavirus emergency has changed every aspect of our everyday lives worldwide. Health care has been affected due to the demand by patients infected with the virus not only in the direct medical care they require but also in the consumption of resources that unexpectedly reached high demand, as a result of the extensive number of infected individuals requiring hospitalization. Standard health care has suffered for patients with acute and chronic illnesses, that is, cancer, cardiopathies, and chronic renal diseases, to name a few. The economy of every country has suffered as a result of the quarantine and lack of mobility by the citizens, and the medical science was not spared. There is the need to report clinical and laboratory findings of an unknown disease, find quick and potentially effective solutions against the virus, and transmit them to the scientific community. This has led to a flood of information from different scientific sources and more. Researchers have been pressured or self-pressured to rapidly obtain data in complicated scenarios in acutely ill patients and in an infectious environment, which represents a high risk for the health-care team. Editors and reviewers of medical and/or scientific journals have facilitated the publication of said information with a dangerously fast acceptance speed. Health care workers interested in science, ourselves included, ought to carefully observe these publications in this coronavirus disease
(COVID)-19 era with a critical eye, always searching for scientific evidence and analyzing the methodology of each of these publications.

The Lancet scandal in COVID-19 pandemic times. Is it OK for science to make a mistake?

The world was in awe when The Lancet, one of the most respected scientific journals in medicine, withdrew an article 2 weeks after its publication, questioning the credibility of the data.

The readers, alarmed by a retraction on the treatment of COVID-19 from a journal, which praises itself on having some of the strictest standards in the world of scientific publications, coined it the Lancet scandal. The article reported a high mortality risk among patients with COVID-19 treated with chloroquine (CQ) or hydroxychloroquine (HCQ). The article caused many people to believe that both drugs could have caused more deaths than cures. Research seemed convincing, based on an enormous sample size of around 15,000 patients at 671 hospitals around the world who had been treated with these medications. Yet, it only took days for both the scientists and the public to detect something suspicious, which implicated credibility in the data as well as the institution responsible for data collection and management.

Why did The Lancet withdraw the article, and what can be learned from this?

\section{Correspondence:}

*Francisco J. Bosques Padilla

E-mail: fbosques58@ hotmail.com
Date of reception: 28-07-2020

Date of acceptance: 04-08-2020 DOI: 10.24875/RMU.M20000045
Available online: $30-10-2020$ Medicina Universitaria. 2020;22(3):91-92 www.medicinauniversitaria.org

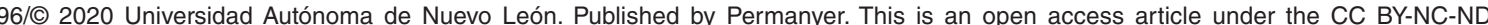
license (http://creativecommons.org/licenses/by-nc-nd/4.0/). 


\section{Matter of integrity and data analysis}

The authors of the article based their research on the analysis of data provided by Surgisphere, a health database management company based in the US. It confirmed having collected medical e-records of over 96,000 patients with COVID-19 in 671 hospitals around the world. It was stated that the registry was automatically updated in real time. At first, the article led the World Health Organization to decide on the solidarity essay research, where over a 100 countries took part in the search for COVID-19 treatments. It also led the governments of the European Union to prohibit the use of both drugs for the treatment of patients with COVID-19.

However, scientists suspected the integrity of the results and the analysis of the data. On May 28, 120 scientists from 24 countries sent a letter to the Lancet raising several questions. Hereafter, we mention only the most relevant regarding the integrity of the science:

1. The authors did not comply with the standard scientific practices of making the raw data available to the general public, despite the fact that the Lancet is one of the signatories of the welcome statement on open access to COVID-19 research data.

2. The article did not mention the specific approval of a research ethics committee, nor did they recognize the contribution of the countries or hospitals where the data were originated. The authors denied the request to divulge the information of the hospitals that provided the data.

3. The daily median dose of $\mathrm{HCQ}$ was $100 \mathrm{mg}$ over that recommended by the United States' Food and Drug Administration, and $66 \%$ of the data was derived from this country.

The Lancet, after receiving this letter, began an independent investigation. A day after this, the article was removed.

\section{What can we learn from this?}

Before an academic journal publishes a scientific manuscript, the journal requests other scientists who work in a similar field to review the article in pairs. The reviewer proves and criticizes the research. As detailed as the review-by-pairs process may be, the reviewers usually do not go into the smallest details of the data without processing, especially if it implicates a large and complex group of data.

This article on the risks of $C Q$ and $H C Q$ proved that the revision by pairs is not completely error-proof. Unfortunately, despite its defects, it is currently the only available way to guarantee scientific rigor before publication.
The "Lancet scandal" showed that data quality is extremely important. The validity of data determines to a large extent the quality and veracity of the analysis. If the obtained data are reliable, it is possible to trust the analysis. In this case, given the doubtful reliability of the source, the analysis and interpretation also become unreliable.

This results in another lesson, the need to boost the so-called open science. Research data should be made available to the general public, which is not a habitual practice among scientific publications yet.

This case also reminds us that a scientific publication of a theory or discovery of a research does not automatically make it a part of science. A research finding is published with the purpose of sharing it to a wider audience, the general public or the scientific community, which can research further. Even though the article from "The Lancet" managed to qualify for a very high standard of publication, it remained open for the public to evaluate its real value even further. In this sense, a theory or discovery can only be accepted as part of science after it holds through a long, exhaustive, and open process or scrutiny and critique. This has occurred for hundreds or even thousands of years in science. In fact, this is the so-called scientific process; scientists are still human who are never fully free of error. There are scientists who have miscalculated; there are scientists who have used an incorrect formula or theoretical concept for a cluster of scientific problems. There are also scientists who have fabricated and falsified their findings. The scientific community has established formal and informal processes to minimize these errors. Unfortunately, these COVID-19 times allow for scientific opportunism.

Formally, the research institutions created scientific committees to examine the scientific value of an investigation and its benefits for humankind. There are also ethics committees, which rigorously examine the compliance of researchers with ethical standards, including, among others, the protection of the participants of a study and the exchange of data. Informally, the real researchers generally appreciate open culture, transparency, and mutual critique. It is considered a part of the maturation process of science. This process is accomplished through scientific publication, as well as interaction in scientific conferences, meetings, or any other means of communication. Thinking and interacting intellectually are essential to solving the world's problems, such as the pandemic, which now surrounds our existence. However, when doing so, we should pay attention to the possibility of making mistakes, which could lead to wrong conclusions. 